\title{
New plasmapause model derived from CHAMP field-aligned current signatures
}

\author{
B. Heilig ${ }^{1,2}$ and H. Lühr ${ }^{1}$ \\ ${ }^{1}$ GFZ German Research Centre for Geosciences, Telegrafenberg, 1473 Potsdam, Germany \\ ${ }^{2}$ Tihany Geophysical Observatory, Geological and Geophysical Institute of Hungary, Kossuth L. u. $91 ., 8237$ Tihany, Hungary \\ Correspondence to: B. Heilig (heilig.balazs@mfgi.hu)
}

Received: 17 December 2012 - Revised: 22 February 2013 - Accepted: 25 February 2013 - Published: 19 March 2013

\begin{abstract}
We introduce a new model for the plasmapause location in the equatorial plane. The determination of the $L$ shell bounding the plasmasphere is based on magnetic field observations made by the CHAMP satellite in the topside ionosphere. Related signals are medium-scale field-aligned currents (MSFAC) (some $10 \mathrm{~km}$ scale size). The mid-latitude boundary of these MSFACs is used for determining the plasmapause. We are presenting a procedure for detecting the MSFAC boundary. Reliable $L$-values are obtained on the night side, whenever the solar zenith angle is below $90^{\circ}$. This means, the boundary is not determined well in the 08:00 to 16:00 magnetic local time (MLT) sector. The radial distance of the boundary is closely controlled by the magnetic activity index Kp. Over the Kp range 0 to 9 , the $L$-value varies from 6 to $2 R_{\mathrm{E}}$. Conversely, the dependence on solar flux is insignificant. For a fixed Kp level, the obtained $L$-values of the boundary form a ring on an MLT dial plot with a centre somewhat offset from the geomagnetic pole. This Kp and local time dependent feature is used for predicting the location of the MSFAC boundary at all MLTs based on a single $L$ value determination by CHAMP. We compared the location of the MSFAC boundary during the years 2001-2002 with the $L$-value of the plasmapause, determined from in situ observations by the IMAGE spacecraft. The mean difference in radial distance is within a $1 R_{\mathrm{E}}$ range for all local times and $\mathrm{Kp}$ values. The plasmapause is generally found earthward of the FAC boundary, except for the duskside. By considering this systematic displacement and by taking into account the diurnal variation and $\mathrm{Kp}$-dependence of the residuals, we are able to construct an empirical model of the plasmapause location that is based on MSFAC measurements from CHAMP. Our new model PPCH-2012 agrees with IMAGE in situ observations within a standard deviation of $0.79 R_{\mathrm{E}}$.
\end{abstract}

Keywords. Magnetospheric physics (Current systems; Plasmasphere)

\section{Introduction}

The plasmapause (PP) is traditionally defined as a sharp density gradient normal to the McIlwain $L$-shell dividing the dense torus-like plasmasphere, co-rotating with the Earth, from the tenuous plasma trough. The plasmasphere consists of cold plasma. Its dynamics are determined predominantly by the electric field. In the plasmasphere the electric field is considered as a superposition of the co-rotational and the dawn-to-dusk electric field. Outside of the PP the solar winddriven convection electric field dominates (Nishida, 1966). According to the classical MHD picture, within the plasmasphere the equipotential surfaces are closed and in a quasisteady state. The PP is the last closed equipotential surface (Brice, 1967). However, under non-steady conditions the last closed equipotential surface is not expected to coincide with the PP.

Soon after its discovery in the early 1960s the first empirical models of the plasmapause appeared (e.g. Binsack, 1967). The frequently cited model of Carpenter and Anderson (1992) was derived from electron density measurements of the ISEE satellites and from electron densities inferred from ground based VLF whistler observations. The model gives the PP location as a function of geomagnetic $\mathrm{Kp}$ indices prevailing some hours before. According to this and later models the PP is more earthward during geomagnetically disturbed periods. O'Brien and Moldwin (2003) used a large dataset of CRESS in situ observations of PP crossings to build their model. They elaborated several versions of the 
PP model depending on the different geomagnetic indices. A new feature in their model is its MLT dependence. The more recent model by Larsen et al. (2007) was the first that is based on solar wind parameters (IMF $B_{\mathrm{Z}}$ and a magnetic merging proxy) instead of some low resolution geomagnetic indices. This model extended the time for a prediction of the PP location, since solar wind parameters are available on the average one hour before they affect magnetospheric processes. The dependence of the above models on the various geomagnetic indices $(\mathrm{Kp}, \mathrm{Dst}, \mathrm{AE})$ and solar wind parameters $\left(B_{\mathrm{Z}}\right.$, merging proxy) indicates that substorm activity, ring current and merging all have some role in the formation of the plasmapause.

The motion of the PP has traditionally been used for estimating the electric field in the inner magnetosphere. For example, during periods of prolonged substorm activity Carpenter et al. (1979) found an outward motion on the duskside and an inward propagation on the dawnside. Recent observations by the IMAGE satellite, in particular, in conjunction with the low altitude DMSP satellites confirmed that the PP motion provides important information about mid-latitude electric fields caused by the solar wind during disturbed periods. Goldstein et al. (2004) used the plasmapause motion in IMAGE EUV images to retrieve information about the electric field in the inner magnetosphere.

Physics-based models of the plasmapause formation were also developed over decades. The more conventional approach utilises an MHD-based convection-only mechanism, where the time variations of the electric field determines dynamically the location of the PP. A comprehensive review on the physics-based plasmasphere models can be found in Pierrard et al. (2009). Lemaire (2001) introduced the kinetic approach in a study of the formation of the PP by taking into account the quasi-interchange instability that is believed to be responsible for the peeling off of the outer plasmasphere shells as a response to the sudden enhancement of magnetospheric convection. Both the convection only but also the interchange-included simulations performed well in reconstructing the PP evolution. In each case the results critically depend on the choice of the $\boldsymbol{E}$-field model (Pierrard et al., 2009).

In the classical picture, the Region 1 field-aligned currents (hereafter R1 FACs) are driven by large-scale magnetospheric plasma convection. At their footprints in the high-latitude ionosphere they set up an electric field distribution according to the conductivity distribution. Towards lower latitudes most of the electric field is shielded by the action of the R2 FACs which are connected to the magnetospheric ring current system. Part of the electric field imprinted by R1 FACs can penetrate, in particular during active periods, to middle and low latitudes (e.g. Kikuchi et al., 1996). This electric field is mapped into the plasmasphere along the highly conducting geomagnetic field lines and influences the dynamics of the plasmapause.
R2 FACs influenced partly (Wang et al., 2005) by the solar wind-driven merging electric field (Kan and Lee, 1979) which acts outside the plasmapause on a global scale. An enhancement of pressure gradients in the storm-time ring current drives downward R2 FACs preferably in the dusk sector. The related electric field causes an erosion of the plasmasphere in the dusk sector driving sunward flowing plumes, and contributes to the dynamics of the PP (Goldstein et al., 2005; Brandt et al., 2005). These events are accompanied by Sub-Auroral Polarisation Streams (SAPS) (Goldstein et al., 2005; Matsui et al., 2009) at ionospheric altitudes.

There is a physical link between the FAC system and the actual plasmapause position. At boundaries where the Alfvén wave velocity is changing abruptly, FACs are generated. According to Kippenhahn and Möllendorf (1975) this can be expressed as

$\frac{\partial j_{\|}}{\partial s}=-\nabla_{\perp} \cdot \frac{\rho}{B_{0}^{2}} \frac{d \boldsymbol{E}}{d t}$,

where $j_{\|}$is the FAC density and $\partial / \partial s$ the change along the field line, $\rho$ is the plasma density, $B_{o}$ the ambient magnetic field and $\boldsymbol{E}$ the electric field. In case of steep density gradient where the electric field changes take place on much larger scales than the density Eq. (1) can be simplified, as described by Lühr et al. (1996)

$\frac{\partial j_{\|}}{\partial s}=-\frac{1}{B_{0}^{2}} \frac{d \boldsymbol{E}}{d t} \cdot \nabla \rho$,

In this case FACs are caused by the component of the electric field changes aligned with the electron density gradient. From the above relations we see that a density gradient focuses FAC activity. When approaching from low $L$-values we argue that the PP is the first boundary where enhanced FAC activity can be expected.

Solar wind-driven intense FACs exist only outside the PP, more precisely outside of the main plasmasphere, i.e. the plasma torus co-rotating with the Earth. On the duskside the bulge contains plasma of plasmaspheric origin. The plasma in the bulge is already detached from the main plasmasphere, but not yet escaped from the magnetosphere. From the observational point of view, the main plasmasphere and the bulge are "essentially two separate entities" (Carpenter et al., 1993). Disturbance associated sunward plasma flows, such as plumes, tails and other structures driven by, for example SAPS electric field, are found outside the R2 FACs. R2 FACs flow between the main plasmasphere and the bulge plasma.

Near and outside the PP not only large-scale (hundreds of $\mathrm{km}$ ) currents, such as R2 FACs, but also medium-scale (few tens of $\mathrm{km}$ ) and small-scale (few $\mathrm{km}$ ) FACs have been observed by CHAMP (Rother et al., 2007).

We found that medium-scale (MS) FACs are commonly observed at high latitudes by CHAMP outside the main plasmasphere, in the region where the solar wind driven electric field is acting. In this paper we make an attempt to monitor the PP position and its motion based on the detection 
of the low-latitude boundary of MSFACs. The direct relation between MSFAC termination and PP latitude has never been investigated before. Magnetic field data from the 10year CHAMP mission are used for this purpose. In addition, making use of in situ electron density measurements of the IMAGE spacecraft, we propose a new empirical model for the plasmapause position.

\section{Data and analysis}

\subsection{CHAMP magnetic data}

The CHAMP satellite was launched on 15 July 2000 into an almost circular, near-polar (inclination $87.3^{\circ}$ ) orbit with an initial altitude of $454 \mathrm{~km}$ which has decreased to $\sim 300 \mathrm{~km}$ after 9 years. An advantage of this orbit is its precession through local time (LT) that makes it possible to investigate the LT dependence of various phenomena. A full local time coverage is achieved in 131 days when considering ascending and descending orbit arcs.

The satellite data used in this study are the pre-processed (level 2) fluxgate magnetometer vector data from CHAMP in sensor frame (product identifier CH-ME-2-FGM-FGM). These are publicly available through the CHAMP Information System and Data Center (http://isdc.gfz-potsdam.de/ champ/). Vector data recorded in the sensor system were transformed into a mean field- aligned (MFA) coordinate system. The mean field was estimated by the CHAMP based field model POMME 4.1s (Potsdam Magnetic Model of the Earth) developed by Maus et al. (2006). This model includes the main field, the crustal anomalies up to spherical harmonic degree/order 90, the field of the ring current, and large-scale magnetospheric fields. To avoid a false interpretation of spatial structures with internal origin as magnetospheric signals resulting from the fast moving satellite through the ambient field, the main and the crustal field were subtracted from the measurements. In the MFA frame the z-component is aligned with the ambient magnetic field direction, the y-component lies in the horizontal plane and is orthogonal to $z$, pointing towards magnetic east. The $\mathrm{x}$-component completes the triad and points outward.

The MFA coordinate system is particularly suitable for distinguishing between ionospheric currents flowing along or across geomagnetic field lines. Our results are presented in "quasi-dipole" latitudes, as defined by the magnetic apex coordinates (Richmond, 1995). $L$-values are calculated using the simple dipole approach:

$$
L=\frac{r}{\cos ^{2} \beta},
$$

where $r$ is the radial distance and $\beta$ the quasi-dipole latitude in apex coordinates of the measurement point.
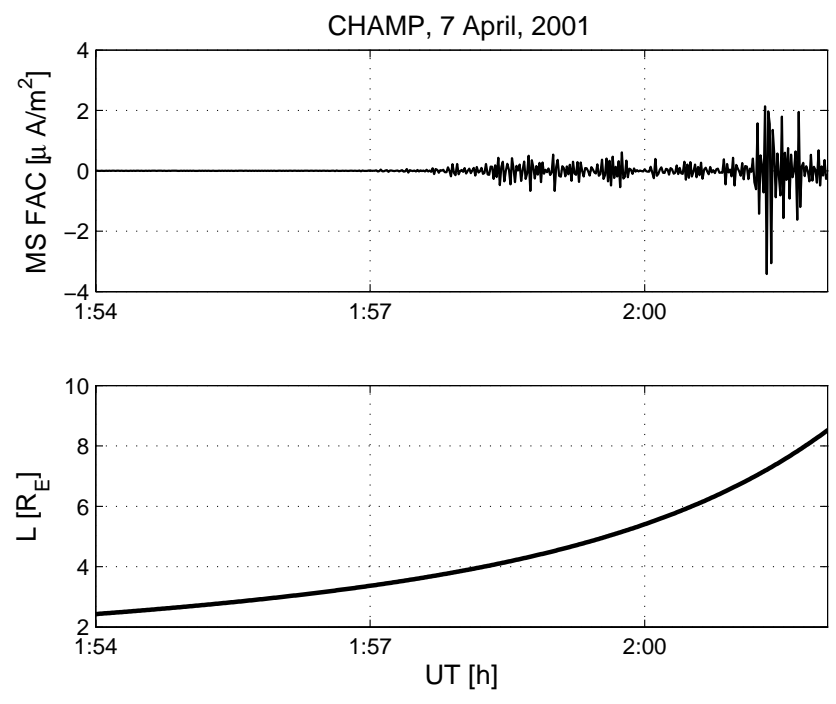

Fig. 1. Example of MSFAC density variation along the orbit. In the bottom panel the $L$-value of the measurements is shown.

\subsection{Detection method}

Our PP model is based on the determination of the midlatitude boundary down to which the magnetic signatures of solar wind driven MSFACs can be observed.

The medium-scale spatial fluctuations correspond to time variations of a few seconds, taking into account the orbital speed of $7.6 \mathrm{~km} \mathrm{~s}^{-1}$ of CHAMP. MSFACs cause significant magnetic signatures in the transverse (toroidal and poloidal) components. In general, the shorter the transverse wavelength of a FAC the larger the current density will be (Ritter and Lühr, 2006, Fig. 10). The other advantage of using the intense MSFACs instead of large-scale FACs is that they have sharper spatial gradient at the PP. We therefore focus on the shorter period variations of the toroidal component.

We have developed an empirical approach for detecting the MSFAC boundary. A characteristic signal, $S$, representing the MSFAC activity is derived through the following steps. First, the toroidal component is filtered using a 3rd order Butterworth high-pass filter with $250 \mathrm{mHz}$ cutoff frequency (corresponds to $30 \mathrm{~km}$ wavelength). Then the MSFAC density is computed as

$j_{\|}=-\frac{1}{\mu_{0} v_{\mathrm{x}}} \frac{d B_{\Phi}}{d t}$,

where $B_{\Phi}$ is the filtered toroidal component, $v_{\mathrm{x}}$ is the orbital speed of the satellite (Ritter and Lühr, 2006).

The upper panel of Fig. 1 shows an example of calculated MSFAC density (observed on 7 April 2001). The lower panel reflects the variation of the $L$-value along the CHAMP orbit. Sizeable MSFAC appear between 01:57-02:00 UT, i.e. between $L=3.5-5.5 R_{\mathrm{E}}$.

As a next step, the logarithm of the squared FAC density in units of $\mu \mathrm{A} \mathrm{m}^{-2}$ was taken, and finally a boxcar averaging 


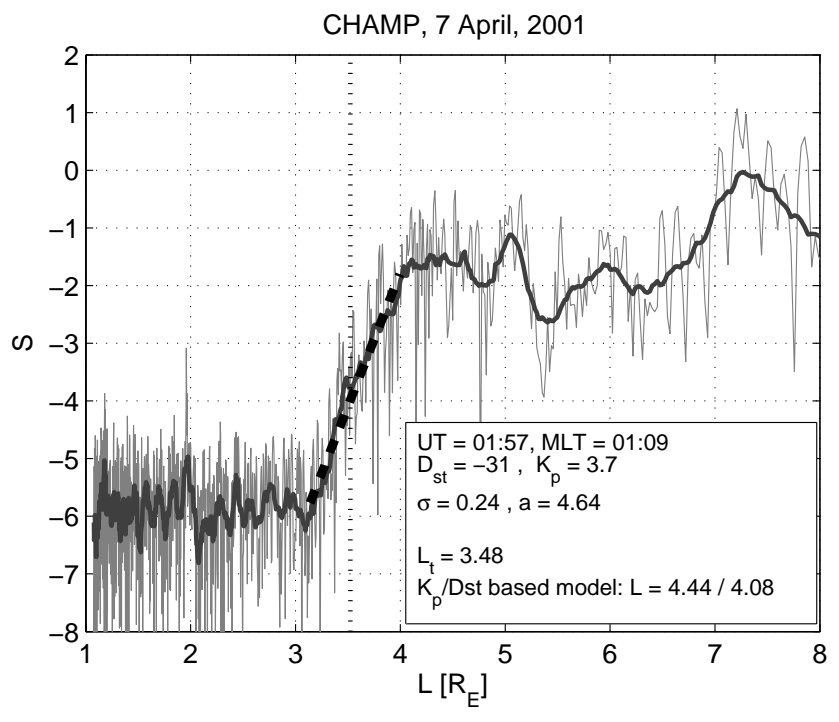

Fig. 2. Well-defined transition of MSFAC activity (data from Fig. 1).

over a 20 s window length was applied to derive $S$ :

$S=\left\langle\log _{10} j_{\|}^{2}\right\rangle_{20 \mathrm{~s}}$.

The signal $S$ was calculated individually for the four high latitude orbital segments of all the orbits. Then these segments were subsequently scanned for steep gradients.

In our procedure for determining the position of PP first $L_{\mathrm{c}}$, the lowest $L$-value ( $>1.5$ ) where $S$ surpasses -2 , is determined.

$L_{\mathrm{c}}=\min (L), S(L)>-2, L>1.5$

Then $L_{\mathrm{m}}$, the highest $L$-value below $L_{\mathrm{c}}$ where $S$ is less than -6 is chosen.

$L_{\mathrm{m}}=\max (L), L<L_{\mathrm{c}}, S(L)<-6$

The applied reference levels of $S$, namely -2 and -6 (corresponding to $10^{-1}$ and $10^{-3} \mu \mathrm{A} \mathrm{m}^{-2}$ (RMS) MSFAC density, respectively) were deduced from a statistical analysis of hundreds of night side satellite passes. Such levels of $S$ are found typically only outside and inside of the nominal PP. The nominal PP positions were calculated according to the model of O'Brien and Moldwin (2003) (hereafter OM2003 model).

The transition of MSFAC amplitude between these two levels can be very different depending on the actual activity conditions. To characterise the transition zone of MSFACs additional quality parameters are introduced. The most important one is $a$, the slope of the best fit straight line

$S^{*}(L)=a L+b$,

which is fitted to the curve $S$ in the interval $\left[L_{\mathrm{m}} ; L_{\mathrm{c}}\right]$, reflecting the sharpness of the boundary. Furthermore, the parameter $\sigma$, the RMS-value of $S(L)-S^{*}(L)$ in the same interval,

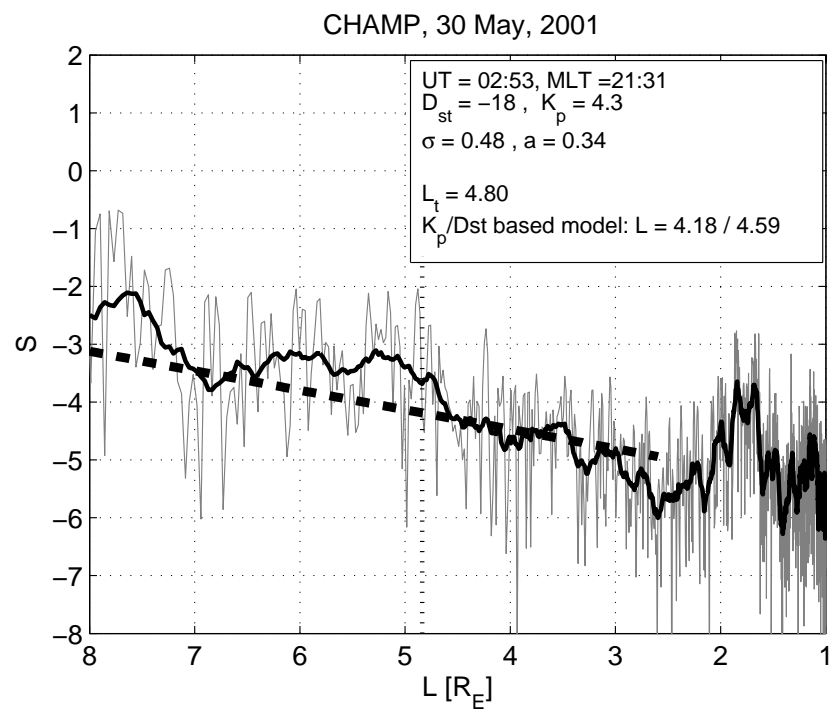

Fig. 3. Poorly definite transition of MSFAC activity.

characterises the quality of the linear fit to $S$. The smaller $\sigma$ is the better the fit:

$\sigma=\sqrt{\left\langle\left(S(L)-S^{*}(L)\right)^{2}\right\rangle}$,

where $L$ is in the interval $\left[L_{\mathrm{m}} ; L_{\mathrm{c}}\right]$. Finally, the transition point, $L_{\mathrm{t}}$ is calculated from the linear function (Eq. 3) at $S^{*}=-3.8 . L_{\mathrm{t}}$ is considered as the equatorward boundary of intense MSFACs:

$L_{\mathrm{t}}=(-3.8-b) / a$.

This reference value was chosen posteriorly based on an analysis to find the best correlation between $L_{\mathrm{t}}$-values and the geomagnetic $\mathrm{Kp}$ index.

Figure 2 illustrates for an actual example the $L_{\mathrm{t}}$ detection procedure (the same data as used for Fig. 1). In this case CHAMP crossed the PP under moderately disturbed conditions on 7 April 2001 at 01:57 UT. The solid grey/black line represents the unsmoothed/smoothed detection signal, $S$ versus the $L$-value. The linear fit, $S^{*}$ is depicted as a dashed black line in the interval $\left[L_{\mathrm{m}} ; L_{\mathrm{c}}\right]$. The MSFAC boundary found at $L_{\mathrm{t}}=3.48 R_{\mathrm{E}}$ is marked by a dotted vertical line. The quality parameters for this case are $a=4.64$ and $\sigma=0.24$. For comparison we also list the model values of the PP position calculated from both the Kp and Dst based versions of the OM2003 model. The Kp(Dst) value to be used as an input for the model is the maximum (minimum) value in the time interval $\left[t_{0}-36 \mathrm{~h} ; t_{0}-2 \mathrm{~h}\right]\left(\left[t_{0}-24 ; t_{0}\right]\right)$, where $t_{0}$ is the time of the observation in hours. Both models yielded higher value $\left(L_{\mathrm{pp}}(\mathrm{Kp})=4.44 R_{\mathrm{E}}, L_{\mathrm{PP}}\left(D_{\mathrm{ST}}\right)=4.08 R_{\mathrm{E}}\right)$ than $L_{\mathrm{t}}$.

Figure 3 represents a poorer example without a definite transition. In this case $a$ is rather low (0.34) and $\sigma$ is significantly higher $(0.48)$ than in the previous example. Here the model values (4.18/4.59) are lower than $L_{\mathrm{t}}(4.80)$. The low 
gradient (low $a$ ) implies a larger uncertainty in the determination of the FAC boundary. The effect of the gradient on precision of the method will be addressed later in the Discussion section.

Both examples presented are from the night side (MLT: 01:09, 21:31). Our experience is that in this time sector our approach works, in general, reliably, as we will demonstrate in the Discussion section.

\section{Observations}

For studying the characteristics of the MSFAC boundary and for the development and validation of the new PP model we have used CHAMP observations of the years 2001-2003. During these years in total 28681 night side (solar zenith angle, $\chi>90^{\circ}$ ) PP crossings were analysed in this way. For a dedicated analysis we selected night side crossings with sharp $(a>0.5)$ transitions. Extremes $(a \geq 10)$ were also excluded. These conditions reduced the dataset down to 24309 cases, which is $85 \%$ of all night side crossings. To remove outliers in the data series, we compared every individual observation with a 5-point boxcar averaged values. Observations with more than $0.5 R_{\mathrm{E}}$ deviation were omitted. This yielded overall 20657 night side PP crossings, i.e. about 19 per day on average, allowing for a continuous monitoring of the PP dynamics.

The top panel of Fig. 4 presents the observed $L_{\mathrm{t}}$ variations (dots) for an interval of 45 days (DoY 75-120, 2001). For comparison, in the 2 nd panel we plotted the $L_{\mathrm{pp}}$-values (dots) derived from the OM2003 model. This model can be parametrised by geomagnetic indices (Kp, Dst or $\mathrm{AE}$ ) and MLT. The version considered here is the one depending on $\mathrm{Kp}$ and MLT. Kp and MLT variations are also shown in the 3rd and bottom panels of the same figure. It is obvious even from the figure that the correlation between $\mathrm{Kp}$ and $L_{\mathrm{t}}$ is stronger, than between $L_{\mathrm{OM} 2003}$ and $L_{\mathrm{t}}$. Small details of Kp variation clearly appear in the temporal evolution of $L_{\mathrm{t}}$. Open circles in the upper two panels depict PP positions derived from IMAGE in situ observations that will be discussed later. The MLTs of IMAGE observations are also shown in the bottom panel as open circles. They agree very well with the time zone of CHAMP measurements.

The observed transition latitude, $L_{\mathrm{t}}$, clearly follows the variation of the modelled PP position, $L_{\mathrm{OM} 2003}$. The correlation coefficient between the observed $L_{\mathrm{t}} \mathrm{S}$ and modelled PP loci is 0.73 for the Kp-based version of the OM2003 model and 0.63 for the Dst version for the 45-day period considered. The correlation coefficients for 2001 are 0.62 and 0.53 for Kp and Dst based models, respectively. Since the correlation with the Kp based model was always found to be higher, we used this model version in Fig. 4 and for further analysis. Note that only night side observations were considered in the above correlation analysis.

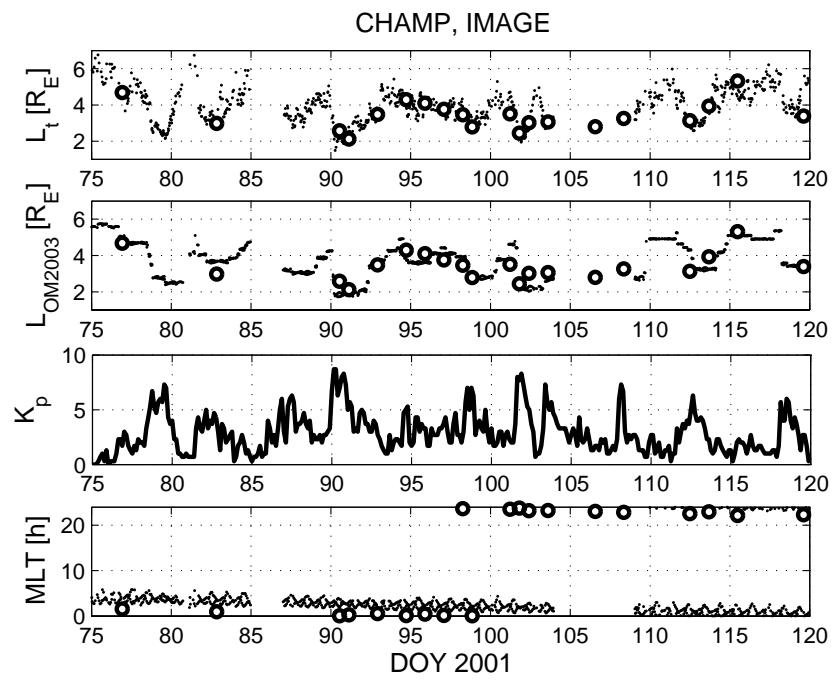

Fig. 4. Variation of plasmapause position. Top panel: The observed $L_{\mathrm{t}}$ (for $0.5<a<10, \chi>90^{\circ}$ ), 2nd panel: the model PP positions, $L_{\mathrm{OM} 2003}$, 3rd panel: $K_{p}$ index, bottom panel: MLT of CHAMP orbit.

\section{Discussion}

In the previous sections we have presented our approach for estimating the plasmapause position from field-aligned current measurements. Here we try to assess the reliability of the $L_{\mathrm{t}}$ determination and compare the results with other models and observations. Finally, we are proposing a new empirical model for the PP location.

\subsection{Correlation analysis}

Since the correlation of $L_{\mathrm{t}}$ with Kp proved to be stronger than with the model PP loci, and because the OM2003 model itself is also Kp dependent, in the following we first analysed the connection with $\mathrm{Kp}$.

Figure 5 summarises the results of our correlation analysis between the CHAMP observed $L_{\mathrm{t}}$ and the Kp index. It shows the correlation coefficients (along with their $95 \%$ confidence intervals) as a function of linear fit (slope $a$, RMS $\sigma$ ), the solar zenith angle $\chi$ and MLT. On the night side the correlation was stronger for crossings with steeper transitions. Figure 5a and $\mathrm{b}$ demonstrate that the correlation coefficient, $c c$, was higher than 0.6 when the slope was larger than 1 , or when $\sigma<0.5$. Moreover, for small slopes the $a$ and $\sigma$ parameters are strongly inter-related. Under the condition $0.5<a<10$, however, $c c$ becomes independent of $\sigma$, and its typical value is above 0.7 . For that reason the value of $\sigma$ was not considered any further as selection parameter.

Figure $5 \mathrm{c}$ and $\mathrm{d}$ show solar zenith angle and MLT dependences from the same correlation analysis (condition: $0.5<$ $a<10$ ). Both curves testify a strong correlation at nighttime; $c c$ surpasses 0.6 between 18 and 07:00 MLT. In Fig. 5c there appears a sudden change in the correlation quality at 

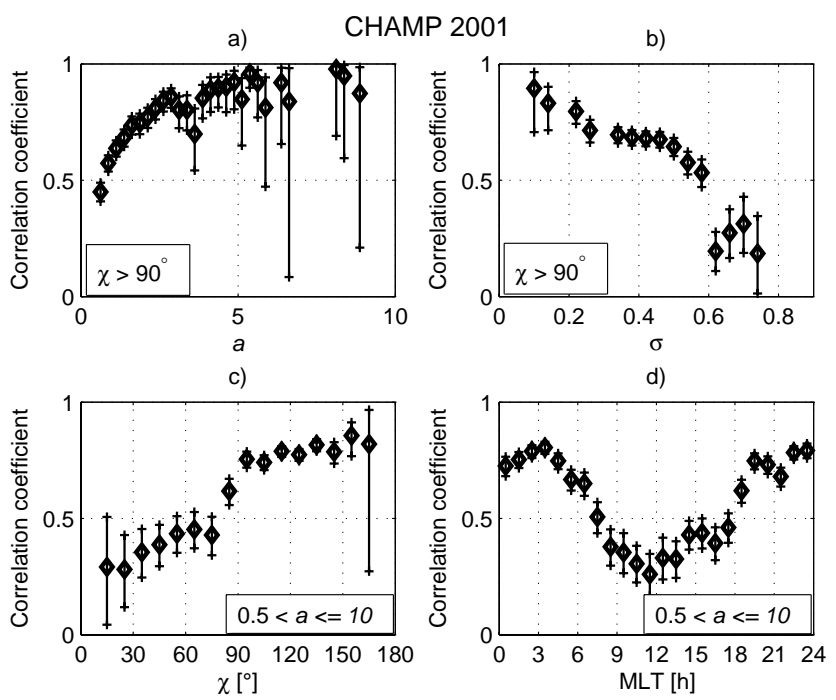

Fig. 5. Dependence of the correlation coefficient between $L_{\mathrm{t}}$ and the Kp index (a) on the slope of the linear fit, $a$, (b) on the standard deviation, $\sigma,(\mathbf{c})$ on the solar zenith angle, $\chi$, and (d) on the magnetic local time, MLT.

sunset/sunrise $\left(\chi=90^{\circ}\right)$. While on the day side $c c<0.5$, on the night side $c c$ is more than 0.7. Based on this result the limit in solar zenith angle, $\chi=90^{\circ}$, was used to distinguish day side and night side observations. The results also clearly show that the PP footprint determination based on the low latitude boundary of MSFACs is reliable only on the night side.

Figure 6 informs about further results of the correlation analysis. The correlation is strongest when $\mathrm{Kp}$ is delayed by one hour with respect to the time CHAMP crosses $L_{\mathrm{t}}$. It should be noted that we used as time assigned to a $\mathrm{Kp}$-value the mid-point of the 3-h interval. By means of a sensitivity study we determined the optimal reference level of $S^{*}$. From the trade-off curve shown in Fig. 6b we deduce $S^{*}=-3.8$ for determining $L_{\mathrm{t}}$ according to Eq. (2). In the sensitivity study the one-hour time delay was taken into account. The optimal reference level was used for the calculation of $L_{\mathrm{t}}$ throughout the analysis.

Figure 7 demonstrates the strong relation between $L_{\mathrm{t}}$ and $\mathrm{Kp}$. At all Kp levels the derived $L_{\mathrm{t}}$ values vary only over a small range. This has motivated us to fit a quadratic regression curve to the mean $L_{\mathrm{t}}$ values:

$L_{\mathrm{t}}=5.726-0.617 \mathrm{Kp}+0.0237 \mathrm{Kp}^{2}$.

For a given $\mathrm{Kp}$ range mean $L_{\mathrm{t}}$ depends only slightly on MLT as shown in Fig. 8. We found no indication of a duskside bulge for the MSFAC boundary, which is often deduced from plasmasphere observations in the equatorial plane (e.g. O'Brien and Moldwin, 2003). Our observations show that $L_{\mathrm{t}}$ extends further to low latitudes on the nightside than on the dayside.
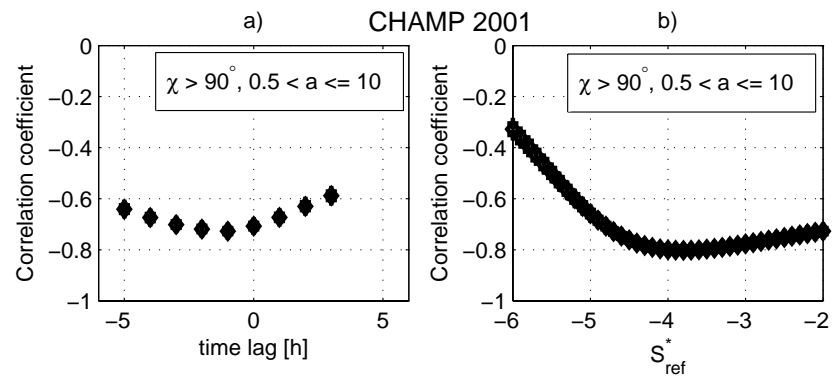

Fig. 6. Dependence of the correlation between $L_{\mathrm{t}}$ and the Kp index on (a) the time lag between $L_{\mathrm{t}}$ and $\mathrm{Kp}$, and (b) on the reference level chosen for $S^{*}$.

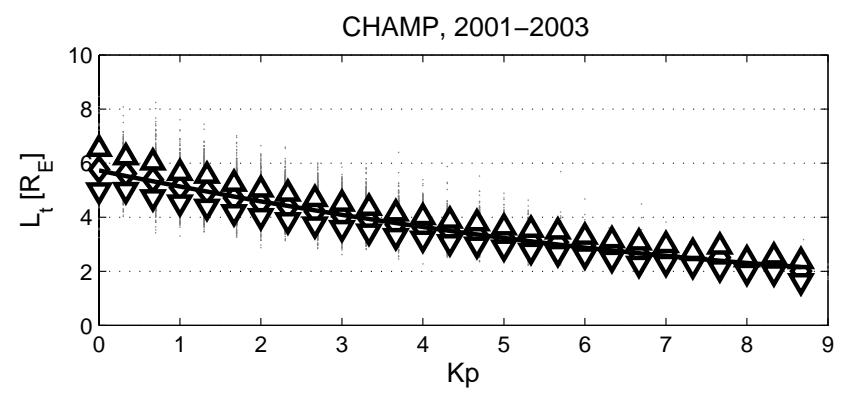

Fig. 7. Dependence of $L_{\mathrm{t}}$ on $\mathrm{Kp}$ (diamonds). Triangles depict mean \pm standard deviations.

From Fig. 8 it is evident that for a given Kp activity level $L_{\mathrm{t}}$ follows almost circles on the dial plot. The origins of the circles, however, are somewhat offset from the centre. In order to investigate this characteristic more qualitatively, we go beyond Eq. (3) and fitted a function to the observed $L_{\mathrm{t}}$ values that depends both on Kp and local time, MLT. The fitting function is similar to that presented by O'Brien and Moldwin (2003)

$$
\begin{aligned}
& L_{\mathrm{t}}=b\left(1+b_{\mathrm{mlt}} \cos \left(\phi-b_{\phi}\right)\right)+\left(a_{1} \cdot \mathrm{Kp}+a_{2} \cdot \mathrm{Kp}^{2}\right) \\
& \quad \cdot\left(1+a_{\mathrm{mlt}} \cos \left(\phi-a_{\phi}\right)\right),
\end{aligned}
$$

where $\phi=2 \pi(\mathrm{MLT} / 24)$, and the parameters describing the MSFAC model are $a_{1}=-0.657, a_{2}=0.0331, a_{\mathrm{mlt}}=$ $0.1113, a_{\phi}=1.040, b=5.911, b_{\mathrm{mlt}}=0.0469$ and $b_{\phi}=$ 2.439. Resulting curves are presented for various Kp levels in Fig. 9. The RMSE of the overall fit is $0.47 R_{\mathrm{E}}$.

Brace et al. (1974) also found a circularly shaped PP based on in situ electron density measurements of the ISIS1 satellite at about $3000 \mathrm{~km}$ altitude, i.e. at the bottomside of the plasmasphere. Obviously, the mid-latitude PP does not exhibit a detectable duskside bulge. More recently, Pedatella et al. (2010), who elaborated a routine process for the determining the PP based on COSMIC GPS total electron content observations of the mid-latitude trough, also reported on circular boundaries with $\mathrm{Kp}$ dependent radii at ionospheric height. 


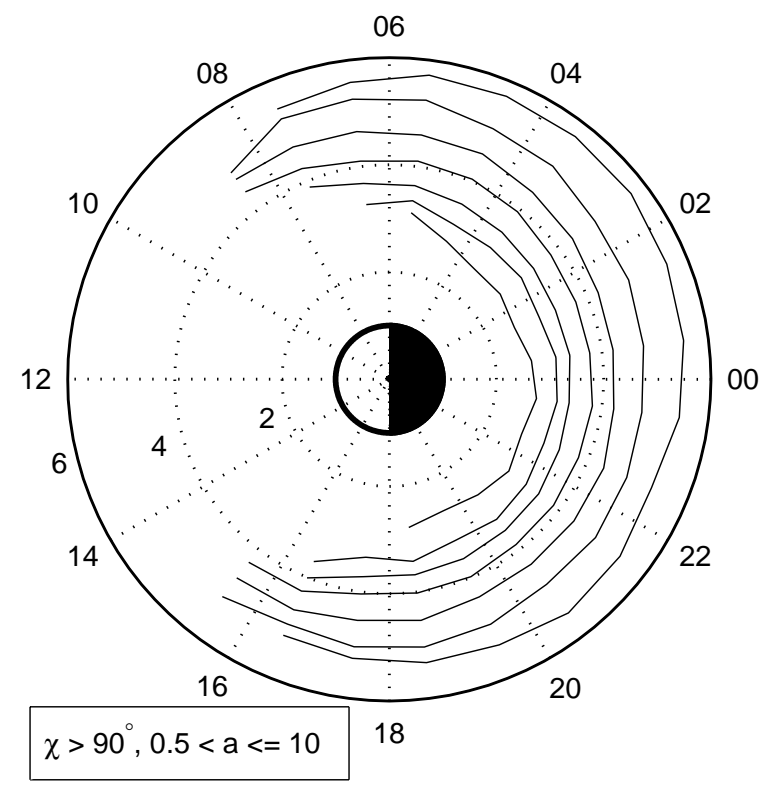

Fig. 8. MLT dependence of mean $L_{\mathrm{t}}$ at different ranges of $\mathrm{Kp}$. Curves from outside inward represent the mean PP positions for $\mathrm{Kp}=0-1,1-2,2-3,3-4,4-5,5-6$, and 6-, respectively. Data from 2001-2003 are used.

Another interesting question is the possible dependence of the plasmapause location on the solar flux level, F10.7. For the analysis we have used only CHAMP measurements from times with $\mathrm{Kp}=2.3$. This ensures a decoupling from the magnetic activity. We found no significant dependence on solar flux.

\subsection{Comparison with IMAGE observations}

Next, we investigated how the low latitude boundary of MSFACs relates to the PP positions. Motivated by the close control of the $L_{\mathrm{t}}$ boundary by the Kp index (Fig. 7) and the particular distribution in local time (Fig. 9), as well as the good agreement of this boundary with IMAGE satellite in situ detections of the plasmapause (Fig. 4), we propose an alternative PP model based on CHAMP field-aligned current measurements.

Before designing the procedure for a model we want to learn more about the relation of the MSFAC boundary to the plasmapause. For that purpose we first estimate $L_{\mathrm{t} 1}$ at any desirable MLT $_{1}$ making use of the circular properties of the MSFAC boundary (see Fig. 9), starting from the actual $L_{\mathrm{t} 0}$ observed at $\mathrm{MLT}_{0}$. It is a two-step procedure. First the actual bias value $b^{\prime}$, the parameter representing the $L$-shell of the MSFAC boundary for $\mathrm{Kp}=0$, is calculated with the help of the MSFAC boundary model represented by Eq. (6).

$b^{\prime}=\frac{L_{t 0}-\left(a_{1} \mathrm{Kp}+a_{2} \mathrm{Kp}^{2}\right)\left(1+a_{\mathrm{mlt}} \cos \left(\phi_{0}-a_{\phi}\right)\right)}{1+b_{\mathrm{mlt}} \cos \left(\phi_{0}-b_{\phi}\right)}$,

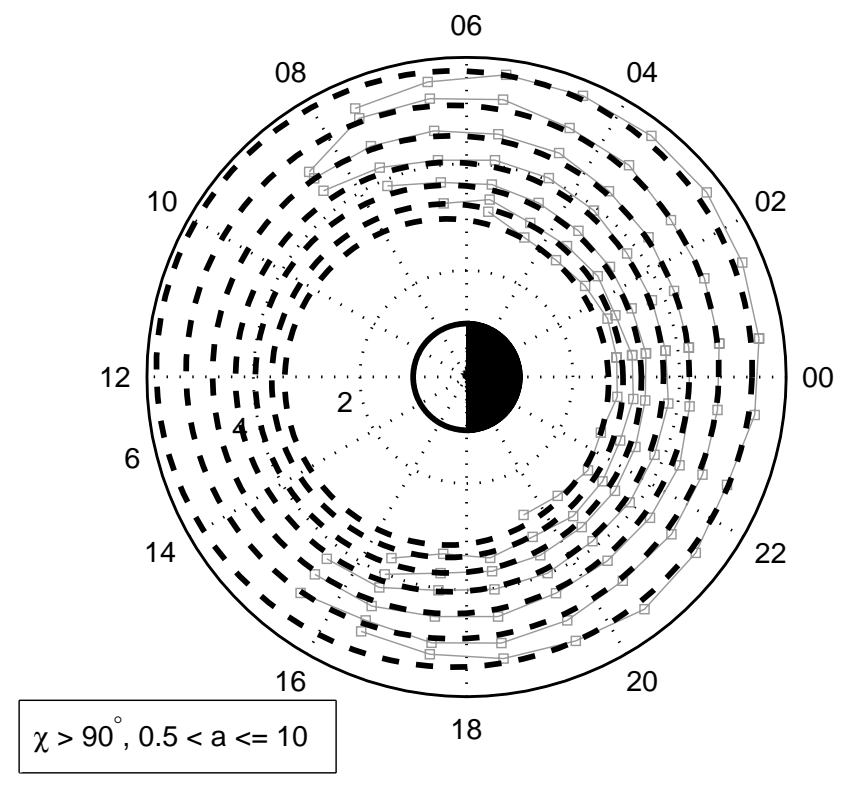

Fig. 9. Average model of the MSFAC boundary as a function of Kp $(\mathrm{Kp}=0.5,1.5,2.5, \ldots 6.5)$ and MLT.

where $L_{\mathrm{t} 0}$ is the actually observed position of MSFAC at $\mathrm{MLT}_{0}$ from which $\phi_{0}$ is determined.

In the second step, $L_{\mathrm{t} 1}$ is calculated by the direct use of Eq. (6) but now with the adjusted $b^{\prime}$ parameter.

For a general assessment of the $L_{\mathrm{t}}$ position with respect to the plasmapause we perform comparisons with in situ measurements of the IMAGE satellite. The Radio Plasma Imager (RPI) on IMAGE made measurements of the local electron density in the magnetosphere.

IMAGE RPI electron density profiles were downloaded for the years 2000-2005 from the CDAWeb maintained by NASA Goddard Space Flight Centre (http://cdaweb.gsfc. nasa.gov). PP positions were determined from electron density profiles as the innermost sharp density gradient. First the innermost interval was selected, where the electron density dropped more than a factor of 5 within $\Delta L<0.5 R_{\mathrm{E}}$. Then the PP position was identified as the inner edge of the sharpest gradient within this interval (Carpenter and Anderson, 1992). PP positions were selected by a fully automated algorithm, but all density profiles were checked by visual inspections. Less defined, multiple or structured PP crossings were not used. Cases when the PP determination was based only on a few points, or when the perigee of the orbit was at $L>3-4$ (the actual threshold depended on geomagnetic activity) or when the density outside the inferred PP was too high, were also rejected. The PP was detectable in 448 cases for the years 2001 and 2002.

We computed the radial difference between $L_{\mathrm{t}}$ and the in situ observation of the actual PP position for every synchronous $(\Delta t<1 \mathrm{~h})$ IMAGE PP and CHAMP MSFAC crossing from 2001 and 2002. Altogether 352 IMAGE PP 

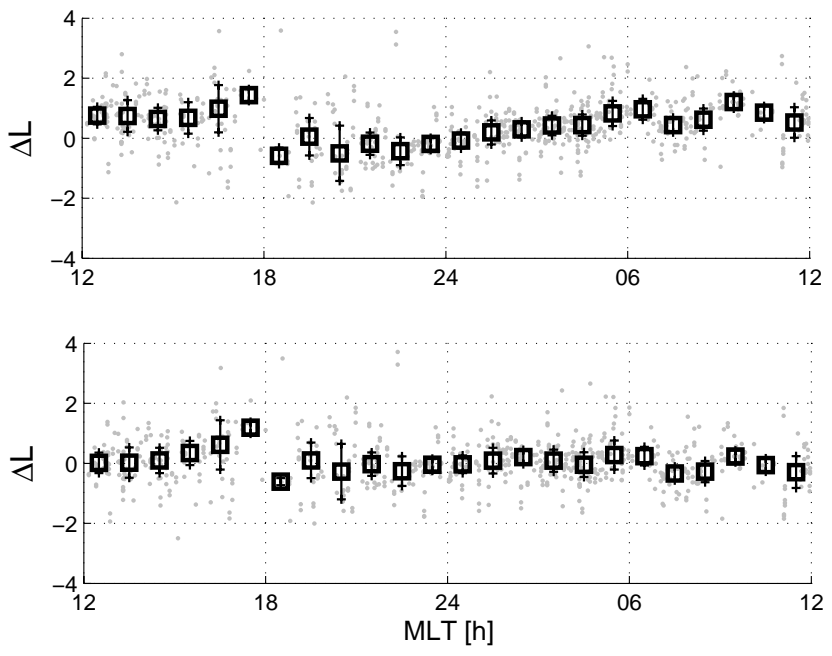

Fig. 10. Comparisons between CHAMP and IMAGE data for the years 2001-2002. Top and bottom panels: Difference between MSFAC boundary and in situ IMAGE RPI observations and PPCH2012 minus IMAGE estimates of PP, respectively.

observations and 731 MSFAC boundary detections were compared. The radial difference was computed as

$\Delta L=L_{\mathrm{t} 1}-L_{\mathrm{PP}-\mathrm{IMAGE}}$

where $L_{\mathrm{t} 1}$ represents predictions from CHAMP at the local time of IMAGE crossings and $L_{\text {PP-IMAGE }}$ the in situ observations. Differences in $L$-values derived from Eq. (8) are presented in the top panel of Fig. 10. $\Delta L$-values are sorted into one-hour bins of magnetic local time. Median values are shown as rectangles; crosses depict the median \pm MAD range. A number of features can be deduced from this figure. Generally, the PP position agrees within $1 R_{\mathrm{E}}$ with the MSFAC boundary. This again confirms the close relation between the two quantities. At closer inspection we find that $\Delta L$ is on average positive, which means that the PP is located about $0.39 R_{\mathrm{E}}$ earthward from $L_{\mathrm{t}}$. We note here that the average spatial resolution of the IMAGE density profiles is $0.18 R_{\mathrm{E}}$ in our dataset, which means that PP positions may have been slightly underestimated. We estimate this systematic error to be less than $0.1 R_{\mathrm{E}}$ on average and always less than $0.24 R_{\mathrm{E}}$. This slight uncertainty is neglected in the development of the model. Furthermore, we find a sinusoidal variation of the residuals, indicating a local time dependence of $\Delta L . \Delta L$-values between $16 \mathrm{~h}$ and $19 \mathrm{~h}$ MLT are considered as outliers represented by only a few observations. They have not been regarded in the further analysis. More discussion on this MLT sector will be given in the next sub-section.

To quantify the difference between CHAMP MSFAC and IMAGE PP observations, we fit a two-dimensional function to the calculated $\Delta L$ values depending on MLT and Kp:

$$
\Delta L=d\left(1+d_{\mathrm{mlt}} \cos \left(\phi-d_{\phi}\right)\right)+e_{\mathrm{mlt}} \cos \left(\phi-e_{\phi}\right) \cdot \mathrm{Kp},
$$

where $\phi=2 \pi \cdot \operatorname{MLT} / 24$, and the other resulting parameters are $d=0.3642, d_{\mathrm{mlt}}=-1.488, d_{\phi}=-0.2547, e_{\mathrm{mlt}}=$ $-0.0665, e_{\phi}=-1.900$. This approximation is based on the activity range $\mathrm{Kp}<6$.

\subsection{Proposal of a plasmapause model}

For the construction of the new PP model we take advantage of the characteristics that we determined in the previous sub-sections about the MSFAC boundary. In particular, the systematic differences in $L$-value between the FAC boundary and the PP location according to IMAGE observations, as depicted in the upper panel of Fig. 10 and quantified in Eq. (9) are taken into account.

Primary input for the model calculation is the value of $L_{\mathrm{t} 0}$ determined by CHAMP at a local time $\mathrm{MLT}_{0}$. There is a 3step procedure foreseen for a prediction of the plasmapause $L_{\mathrm{t} 1}$ at $\mathrm{MLT}_{1}$.

1. Calculation of the adjusted bias parameter $b^{\prime}$ as given in Eq. (7).

2. Calculation of $L_{\mathrm{t} 1}\left(\mathrm{Kp}, \mathrm{MLT}_{1}\right)$ by the direct use of Eq. (6) at any $\mathrm{MLT}_{1}$ with the adjusted $b^{\prime}$ parameter. The results obtained here represent a model of the MSFAC boundary, which may be of interest on its own.

3. Estimation of the PP position taking into account the observed differences between MSFAC and PP from IMAGE,

$$
L_{\mathrm{pp}}\left(\mathrm{Kp} p, \mathrm{MLT}_{1}\right)=L_{\mathrm{t} 1}\left(\mathrm{Kp}, \mathrm{MLT}_{1}\right)-\Delta L\left(\mathrm{Kp}, \mathrm{MLT}_{1}\right),
$$

where $\Delta L$ is the functional value of Eq. (9) for a given $\mathrm{Kp}$ and $\mathrm{MLT}_{1}$.

We term this new empirical model PPCH-2012.

For validating the predictions of the PPCH-2012 model, we took again advantage of all available IMAGE plasma density measurements from PP crossings of the years 2001 and 2002. Radial differences are calculated in the same way as in Eq. (8)

$$
\Delta L^{*}=L_{\mathrm{PPCH}}-L_{\mathrm{PP}-\mathrm{IMAGE}}
$$

Results are plotted in the lower frame of Fig. 10. We find an excellent agreement of our model with IMAGE in situ observations at all MLT hours. Here again, the values between 16:00 and 19:00 MLT stand out. They have not been considered in our modelling procedure. We relate these apparent outliers to the dynamic behaviour of the PP in the duskside bulge region, e.g. to the presence of sub-auroral polarisation streams (SAPS), plumes, etc., and the abrupt westward edge of the bulge as described in the introduction (e.g. Carpenter et al., 1993). SAPS partly overlap with the plasmasphere forming badly disturbed plasmapause shapes (e.g. Foster et al., 2007). 


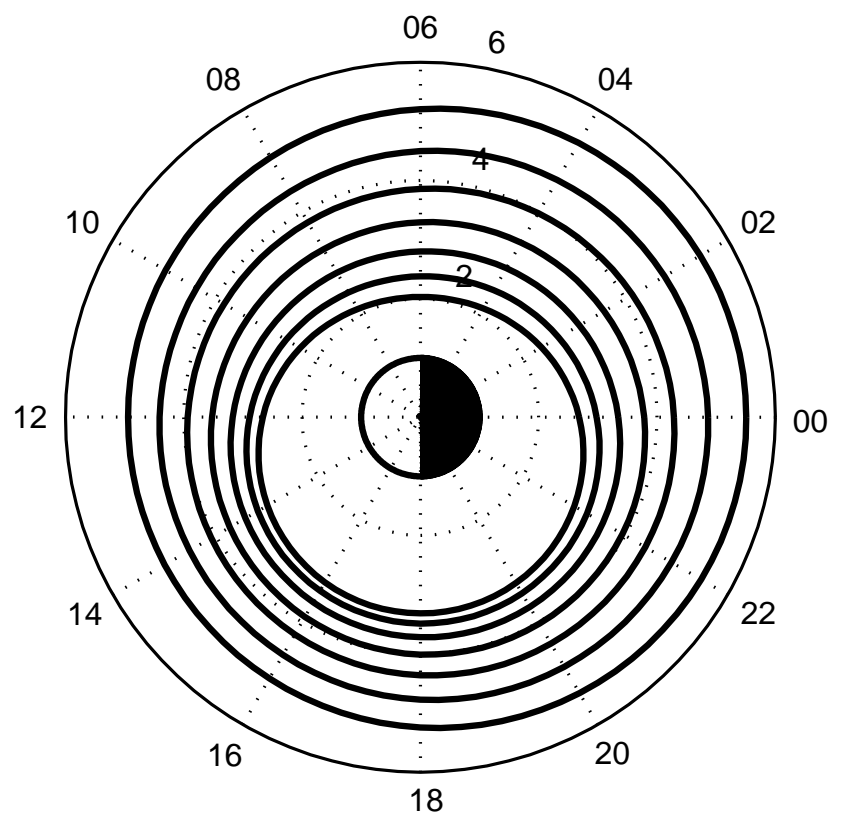

Fig. 11. Average shape of the $\mathrm{PPCH}-2012$ plasmapause position in $L$-value space for $\mathrm{Kp}=0.5,1.5, \ldots 6.5$.

After the very promising results in comparison with IMAGE, we looked a little more into the general features of the PPCH-2012 model. One interesting point is the dependence of the radial distance on magnetic local time. In Fig. 11 we have plotted the diurnal variation of the $L$-values of our PPCH-2012 model for different magnetic activity levels. Here a displacement towards larger $L$-values on the duskside is particularly clear for enhanced magnetic activity. In Fig. 12 the MSFAC boundary and the PPCH-2012 model are compared directly. Different from the MSFAC boundary which exhibits largest $L$-values at noon and smallest at midnight, PPCH-2012 peaks between 18:00 and 19:00 MLT during active periods, thus it nicely reflects the plasmaspheric dusk bulge. Smallest plasmasphere expansions are found in the morning sector. As mentioned in the introduction, R2 FACs can be found, in general, outside the PP. Around noon MSFAC is located about $1 R_{\mathrm{E}}$ outward of the plasmapause. However, when comparing our PP model with MSFAC one has to remember that no reliable FAC locations could be determined during the hours around noon. Those MSFAC positions are just extrapolations resulting from the circular fit. In this noon time sector, the MSFAC location depends on our assumptions.

The R2 FACs surround the main, co-rotating body of the plasmasphere. In the dusk and early night sector MSFACs and PP coincide reasonably well. Here detached plasma or plasma plumes can be found outside the R2 FAC sheet. This is the region where strong SAPS electric fields move the plasma sunward. The formation of the duskside bulge is limited to low latitudes (e.g. Brace et al., 1974). As discussed be-

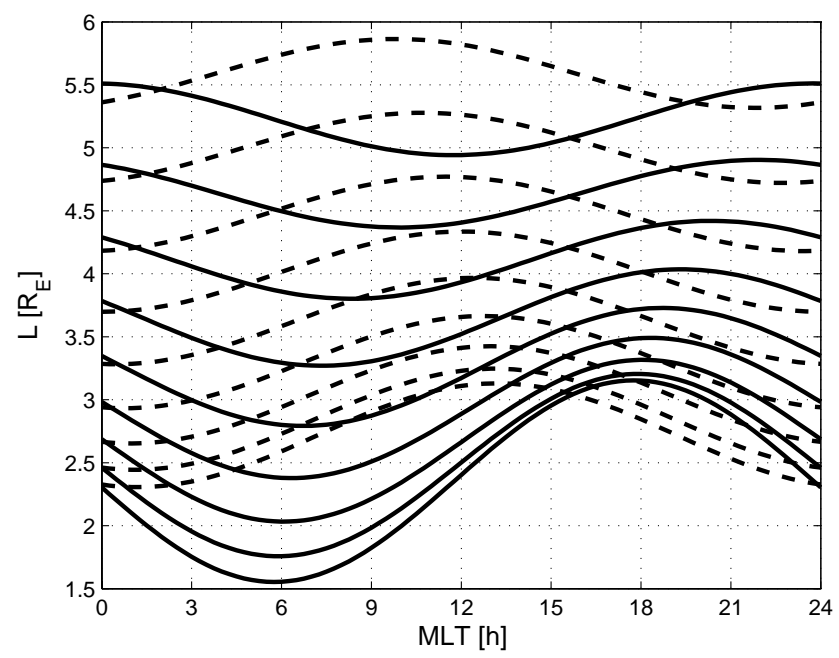

Fig. 12. Comparison of the diurnal variation of the MSFAC boundary (dashed) and the PPCH-2012 plasmapause position (solid) for $\mathrm{Kp}=0.5,1.5, \ldots 8.5$.

fore, at mid-latitudes the $L$-shell coinciding with the PP position hardly depends on local time; here the bulge is missing. This difference in MLT behaviour of MSFAC boundary and PP is reflected in Fig. 12 and in Fig. 10. The MSFAC boundary may closely correspond to the ionospheric projection of the boundary of the main plasmasphere. The R2 FACs seem to flow between the detached plasma of the bulge and the main plasmasphere. Undisputable differences appear during early morning through pre-noon. Here MSFAC is encountered on $L$-values up to $1 R_{\mathrm{E}}$ larger than PP. In that time sector there are obviously no electric field variations just outside the plasmapause that could drive FACs. Further investigations, for example by the two Van Allen spacecraft, may be needed for resolving that question.

PPCH-2012 was also compared with the OM2003 model (see Fig. 13). The two models show a lot of similarities. Both exhibit a bulge whose maximum moves in MLT with $\mathrm{Kp}$ toward earlier times. At low geomagnetic activity both models predict the smallest PP distance on the dayside, and the largest around midnight. However, the locations of the boundary, the range of the diurnal variations, as well as the dependence on $\mathrm{Kp}$ is somewhat different. $\mathrm{PPCH}-2012$ reflects the changes in geomagnetic activity $(\mathrm{Kp})$ more prominent than the OM2003 model. Hence, PPCH-2012 reflects more details of the PP dynamics. This is expected since it is updated continuously by actual MSFAC observations.

For the application of the PPCH-2012 model actual values of $L_{\mathrm{t} 0}$ at $\mathrm{MLT}_{0}$ from CHAMP are required. These are calculated for the whole mission, August 2000 to August 2010. When visiting the web site http://www.gfz-potsdam.de/ CHAMP_currents a brief description of the PPCH-2012 model can be found and a link to a file with the values for the MSFAC boundary. The reliability of PPCH-2012 predictions 


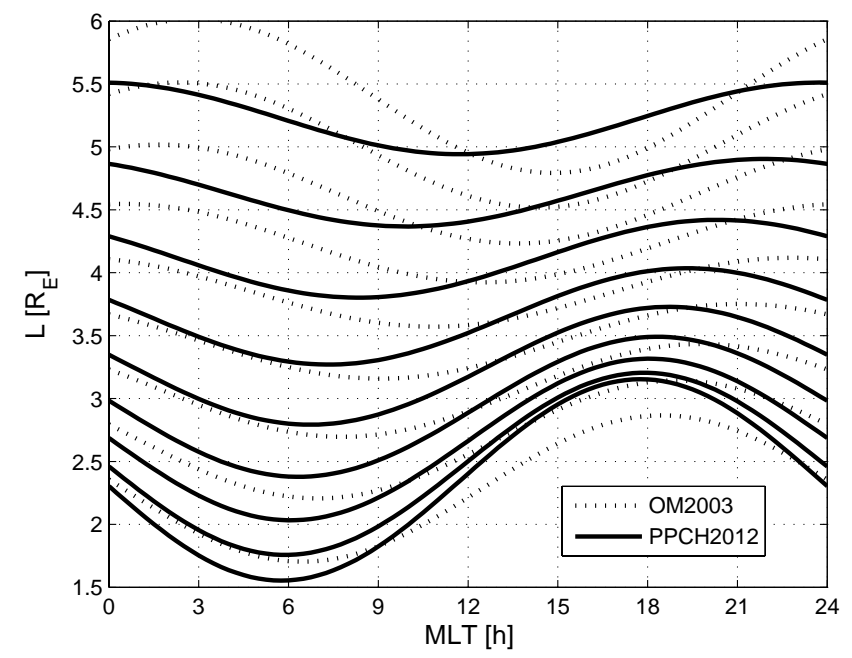

Fig. 13. Diurnal variation of the PP radial distance from the PPCH2012 model (solid) and the OM2003 model (dotted) at $\mathrm{Kp}=0.5,1.5$, ... 8.5. For details see text.

may be reduced for hours around noon (09:00-15:00 MLT) since no direct MSFAC observations are available from that sector. For times where no CHAMP data are available one may use PPCH-2012 with reduced accuracy by relying on the correlation with $\mathrm{Kp}$. In that case the processing starts with Eq. (6), and follows the same approach.

\section{Conclusions}

We have proposed a new empirical model of the plasmapause position (termed PPCH-2012) based on field-aligned current measurements by the CHAMP satellite. A specific approach is described for reliably detecting the related boundary of FAC activity. Some important features of this boundary have been deduced.

1. The mid-latitude boundary of medium-scale fieldaligned currents (MSFAC) is closely related to the location of the plasmapause (PP) at all levels of activity. The MSFAC boundary cannot be determined reliably on the dayside (08:00 to 16:00 MLT).

2. There is a strong control of the MSFAC radial distance by the magnetic activity index Kp. During enhanced activity the boundary moves inward. Conversely, the solar flux level has no significant influence on the location of MSFAC boundary.

3. For a constant Kp level the MSFAC boundary appears on $L$-values which form a ring around the pole with a centre somewhat offset from the geomagnetic pole. For increasing magnetic activity the rings become smaller. This circular characteristic has been used to predict the location of the MSFAC boundary at all local times.
4. A comparison of the MSFAC boundary with the PP deduced from IMAGE in situ observations revealed an agreement within $1 R_{\mathrm{E}}$ radial distance for all local times and activity levels. The PP is generally found earthward of MSFAC, on average by about $0.39 R_{\mathrm{E}}$, except for the duskside bulge region.

5. An empirical model for the PP location is constructed based on the actual MSFAC measurements of CHAMP and by taking into account the systematic differences in radial distance between the FAC boundary and the PP position as observed by IMAGE. The difference comprises a diurnal variation and a Kp-dependence. The predictions of our new model, PPCH-2012, agree with IMAGE in situ observations within a standard deviation of $0.79 R_{\mathrm{E}}$.

CHAMP has provided updates of the PP location about 19 times a day on average during the years 2000 through 2010. This is regarded as a valuable dataset for studying the dynamics of the PP during various phases of solar and magnetic activities.

Acknowledgements. The research leading to these results has received funding from the European Community's Seventh Framework Programme ([FP7/2007-2013]) under grant agreement number 263218. A large part of the work was performed during a research visit of $\mathrm{B}$. Heilig at GFZ in Potsdam. The CHAMP mission was sponsored by the Space Agency of the German Aerospace Center (DLR) through funds of the Federal Ministry of Economics and Technology, following a decision of the German Federal Parliament (grant code 50EE0944). IMAGE RPI electron density data were acquired through CDAWeb/SPDF. We thank the principal investigators Bodo Reinisch, Philip Webb, and Yongli Wang.

The service charges for this open access publication have been covered by a Research Centre of the Helmholtz Association.

Topical Editor L. Blomberg thanks T. Kikuchi and V. Pierrard for their help in evaluating this paper.

\section{References}

Binsack, J. H.: Plasmapause observations with the M.I.T. experiment on IMP 2, J. Geophys. Res., 72, 5231-5237, 1967.

Brace, L. H. and Theis, R. F.: The Behavior of the Plasmapause at Mid-Latitudes: Isis 1 Langmuir Probe Measurements, J. Geophys. Res., 79, 1871-1884, 1974.

Brandt, P. C., Goldstein, J., Anderson, B. J., Korth, H., Immel, T. J., Roelof, E. C., DeMajistre, R., Mitchell, D. G., and Sandel, B.: On the Relation Between Electric Fields in the Inner Magnetosphere, Ring Current, Auroral Conductance, and Plasmapause Motion, in: Inner Magnetosphere Interactions: New Perspectives From Imaging, edited by: Burch, J., Schulz, M., and Spence, H., 
Geophysical Monograph 159, AGU, Washington D.C., 159-166, 2005.

Brice, N. M.: Bulk Motion of the Magnetosphere, J. Geophys. Res., 72, 5193-5211, 1967.

Carpenter, D. L. and Anderson, R. R.: An ISEE/Whistler model of Equatorial Electron Density in the Magnetosphere, J. Geophys. Res., 97, 1097-1108, 1992.

Carpenter, D. L., Park, C. G., and Miller, T. R.: A model of substorm electric fields in the plasmasphere based on whistler data, J. Geophys. Res., 84, 6559-6563, 1979.

Carpenter, D. L., Giles, B. L., Chappel, C. R., Décréau, P. M. E., Anderson, R.R., Persoon, A. M., Smith, A. J., Corcuff, Y., and Canu, P.: Plasmasphere Dynamics in the Duskside Bulge Region: a New Look at an Old Topic, J. Geophys. Res., 98, 19243-19271, 1993.

Foster, J. C., Rideout, W., Sandel, B., Forrester, W. T., and Rich, F. J.: On the relationship of SAPS to storm-enhanced density. J. Atmos. Sol.-Terr. Phys., 69, 303-313, 2007.

Goldstein, J., Sandel, B. R., and Reiff, P. H.: Electric fields deduced from plasmapause motion in IMAGE EUV images, Geophys. Res. Lett., 31, L01801, doi:10.1029/2003GL018386, 2004.

Goldstein, J., Wolf, R. A., Burch, J., and Sandel, B.: Magnetospheric model of subauroral polarization stream, J. Geophys. Res., 110, A09222, doi:10.1029/2005JA011135, 2005.

Kan, J. R. and Lee, L. C.: Energy coupling function and solar windmagnetosphere dynamo, Geophys. Res. Lett., 6, 577-580, 1979.

Kikuchi, T., Lühr, H., Kitamura, T., Saka, O., and Schlegel, K.: Direct penetration of the polar electric field to the equator during a DP 2 event as detected by the auroral and equatorial magnetometer chains and the EISCAT radar, J. Geophys, Res., 101, 1716117173, 1996.

Kippenhahn, R. and Möllenhoff, C.: Elementare Plasmaphysik, B.I. Wissenschaftsverlag, Mannheim, 1975.

Larsen, B. A., Klumpar, D. M., and Gurgiolo, C.: Correlation between plasmapause position and solar wind parameters, J. Atmos. Sol.-Terr. Phys., 69, 334-340, 2007.

Lemaire, J. F.: The formation of the light-ion-trough and peeling off the plasmasphere, J. Atmos. Sol.-Terr. Phys., 63, 1285-1291, 2001.

Lühr, H., Lockwood, M., Sandholt, P. E., Hansen, T. L., and Moretto, T.: Multi-instrument ground-based observations of a travelling convection vortices event, Ann. Geophys., 14, 162181, doi:10.1007/s00585-996-0162-z, 1996.
Matsui, H., Foster, J. C., Carpenter, D. L., Dandouras, I., Darrouzet, F., De Keyser, J., Gallagher, D. L., Goldstein, J., Puhl-Quinn, P. A., and Vallat, C.: Electric Fields and Magnetic Fields in the Plasmasphere: A Perspective From CLUSTER and IMAGE, Space Sci. Rev., 145, 107-135, doi:10.1007/s11214-008-9471-8, 2009.

Maus, S., Rother, M., Hemant, K., Stolle, C., Lühr, H., Kuvshinov, A., and Olsen, N.: Earth's lithospheric magnetic field determined to spherical harmonic degree 90 from CHAMP satellite measurements, Geophys. J. Int., 164, 319-330, doi:0.1111/j.1365246X.2005.02833.x, 2006.

Nishida, A.: Formation of the plasmapause, or magnetospheric plasma knee, by combined action of magnetospheric convection and plasma escape from the tail, J. Geophys. Res., 71, 56695679, 1966.

O'Brien, T. P. and Moldwin, M. B.: Empirical plasmapause models from magnetic indices, Geophys. Res. Lett., 30, 1152, doi:10.1029/2002GL016007, 2003.

Pedatella, N. M. and Larson, K. M.: Routine determination of the plasmapause based on COSMIC GPS total electron content observations of the midlatitude trough, J. Geophys. Res., 115, A09301, doi:10.1029/2010JA015265, 2010.

Pierrard, V., Goldstein, J., Nicolas, A., Jordanova, V. K., Kotova, G. A., Lemaire, J. F., Liemohn, M. W., and Matsui, H.: Recent progress in physics-based models of the plasmasphere, Space Sci. Rev., 145, 193-229, 2009.

Richmond, A. D.: Ionospheric electrodynamics using magnetic apex coordinates, J. Geomagn. Geoelectr., 47, 191-212, 1995.

Ritter, P. and Lühr, H.: Curl-B technique applied to Swarm constellation for determining field-aligned currents, Earth Planets Space, 58, 463-476, 2006.

Rother, M., Schlegel, K., and Lühr, H.: CHAMP observation of intense kilometer-scale field-aligned currents, evidence for an ionospheric Alfvén resonator, Ann. Geophys., 25, 1603-1615, doi:10.5194/angeo-25-1603-2007, 2007.

Wang, H., Lühr, H., and Ma, S. Y.: Solar zenith angle and merging electric field control of field-aligned currents: A statistical study of the Southern Hemisphere, J. Geophys. Res., 110, A03306, doi:10.1029/2004JA010530, 2005. 